\title{
Qualidade da dieta, risco cardiovascular e estado nutricional de trabalhadores assistidos pelo Programa de Alimentação do Trabalhador
}

\author{
Quality of diet, cardiovascular risk and nutritional status of workers assisted by the Worker's \\ Feeding Program
}

\section{DOI: 10.37111/braspenj.2020351010}

Elisangela de Meira

Fernanda Rebequi

Diana Souza Santos $\mathrm{Vaz}^{2}$

Caryna Eurich Mazur ${ }^{3}$

\section{Unitermos:}

Avaliação nutricional. Consumo de alimentos. Nutrição. Antropometria. Dieta.

\section{Keywords:}

Nutrition assessment. Food consumption. Nutrition. Anthropometry. Diet.

\section{Endereço para correspondência:}

Elisangela de Meira

Universidade Estadual do Centro-Oeste - Nutrição Rua Simeão Varela de Sá, 3 - Vila Carli - Guarapuava, PR, Brasil - CEP 85040-080

E-mail: meira_elisangela@hotmail.com

\section{Submissão}

4 de março de 2020

Aceito para publicação

17 de março de 2020

\begin{abstract}
RESUMO
Introdução: Em decorrência das diversas mudanças ocorridas na alimentação e no estilo de vida das pessoas, nota-se um aumento na prevalência de excesso de peso, e também aumento do risco e desenvolvimento de doenças cardiovasculares (DCV). É importante avaliar a qualidade da alimentação, para conhecê-la e elaborar intervenções adequadas, especialmente aos trabalhadores, uma vez que fazem a maior parte de suas refeições fora de casa, e estão mais propensos para o risco para DCV. O objetivo deste estudo foi avaliar o estado nutricional, a qualidade da dieta e sua relação com DCV, em trabalhadores de uma indústria de carnes. Método: Estudo transversal realizado entre outubro e dezembro de 2017. Foram utilizados formulários, realizada avaliação antropométrica e dietética dos trabalhadores de uma indústria de carnes no município de Pitanga, Paraná. Para cálculo e avaliação do consumo alimentar foi utilizado o software Nutrabem ${ }^{\circledR}$, o qual determinou os valores descritivos do índice de qualidade da dieta (IQD), baseados nos grupos alimentares segundo a pirâmide dos alimentos. O IQD foi dividido em três categorias: de $80 \mathrm{a}$ 100 pontos - dieta saudável, de 50 a 79 pontos - necessita de ajustes no padrão alimentar, menor que 49 pontos - dieta inadequada. Resultados: Foram incluídos no estudo 55 funcionários, com média de idade de $32,2 \pm 9,92$ anos, predominando o sexo masculino $(67,3 \%)$, média de índice de massa corporal de $27,05 \pm 4,90 \mathrm{~kg} / \mathrm{m}^{2}$, com valores superiores no grupo com risco de DCV, conforme a razão cintura-estatura. O IQD médio foi de $52,0 \pm 10,9$ pontos, sendo $40 \%(n=22)$ dos funcionários classificados com dieta inadequada e $60 \%(\mathrm{n}=33)$ com necessidade de ajuste na dieta, as mulheres apresentaram uma dieta inadequada comparado aos homens $(p<0,05)$. Conclusão: Estado nutricional, idade e consumo calórico tiveram relação com a qualidade da dieta e o risco para DCV, é importante o acompanhamento nutricional e ações de intervenção com a classe trabalhadora.
\end{abstract}

\section{ABSTRACT}

Introduction: Due to the various changes that have occurred in people's diet and lifestyle, there is an increase in the prevalence of overweight, as well as an increased risk and development of cardiovascular disease (CVD). It is important to assess the quality of food, to know it and to develop appropriate interventions, especially for workers, since they eat most of their meals away from home and are at risk for CVD. The objective of this study was to evaluate the nutritional status, the quality of the diet and its relationship with CVD in meat industry workers. Methods: Crosssectional study conducted from October to December 2017. Forms were used, anthropometric and dietary evaluation of workers of a meat industry in the municipality of Pitanga, Paraná, Brazil. Nutrabem ${ }^{\circledR}$ software was used to calculate and evaluate food consumption, which determined the descriptive values of the diet quality index (IQD), based on the food groups according to the food pyramid. The IQD was divided into three categories: 80 to 100 points - healthy diet, 50 to 79 points - needs adjustments in the eating pattern, less than 49 points inadequate diet. Results: 55 employees were included with a mean age of $32.2 \pm 9.92$ years, predominantly male $(67.3 \%)$ average body mass index of $27.05 \pm 4.90 \mathrm{~kg} / \mathrm{m}^{2}$, with values in the group at risk of CVD according to waist-to-height ratio. The average IQD was $52.0 \pm 10.9$ points, with $40 \%(n=22)$ of employees classified with inadequate diet and $60 \%(n=33)$ needing adjustment in diet, women showed a tendency to diet. inadequate compared to men $(p<0.05)$. Conclusion: Nutritional status, age and caloric intake was related to the quality of the diet and the risk for CVD. It is important the nutritional monitoring and intervention actions with the working class.

1. Nutricionista, Pós-graduação em Nutrição Clínica - Centro Universitário Campo Real, Guarapuava, PR, Brasil.

2. Nutricionista, Programa de Pós-graduação em Ciências da Saúde - Universidade Estadual de Campinas, Campinas, SP, Brasil.

3. Nutricionista, Docente Universidade Estadual do Centro-Oeste, Guarapuava, PR, Brasil. 


\section{INTRODUÇÃO}

Em decorrência de mudanças ocorridas devido à globalização, no que diz respeito ao estilo de vida das pessoas e de seus hábitos alimentares, nota-se elevado consumo de alimentos ricos em gorduras, açúcares e alimentos refinados, reduzida ingestão de carboidratos complexos, fibras e minerais, evidenciando o processo de transição nutricional ocorrida desde o último século, em diferentes países. $\bigcirc$ que se nota é o aumento gradativo em relação à dieta da população em geral, e que a mesma vem se tornando extremamente monótona no que tange à variedade, e não sustentável' ${ }^{1}$. Esses fatores, aliados ao declínio da atividade física, resultam em alterações na composição corporal, aumentando a prevalência de excesso de peso, predispondo o desenvolvimento de doenças crônicas não transmissíveis (DCNT), como doenças cardiovasculares, diabetes e certos tipos de câncer ${ }^{2}$. Juntamente com isso, por não ser uma dieta sustentável, além de doenças devido ao excesso, teremos cada vez mais desnutrição e um meio ambiente gravemente degradado ${ }^{3}$.

A Organização Mundial da Saúde (OMS) estima que, em 2030 , quase 23,6 milhões de pessoas podem morrer devido às doenças cardiovasculares (DCV), responsável por duas de cada três mortes no mundo ${ }^{4}$. É grande a preocupação que a prevalência de seus fatores de risco, sobrepeso e obesidade vem aumentando consideravelmente. Em 2015, 603,7 milhões de adultos eram obesos (IMC $\left.\geq 30 \mathrm{~kg} / \mathrm{m}^{2}\right)^{5}$. No Brasil, 50,8\% da população estavam acima do peso, sendo $17,5 \%$ considerados obesos, em 20136,7.

método mais utilizado em coletividades para definir sobrepeso e obesidade tem sido o índice de massa corporal (IMC), permitindo avaliar e diagnosticar indivíduos com maiores chances de desenvolver DCV. Todavia, simplificadamente, a obesidade caracteriza-se pelo acúmulo de gordura no organismo e a distribuição dessa gordura tem relação direta com o risco de morbimortalidade. A circunferência da cintura (CC) é o método utilizado para determinar risco para DCV e obesidade, combinado ao IMC, a CC tem sido reconhecida como preditor para risco de morbidade ${ }^{8,9}$.

Recentemente, a razão cintura-estatura (RCE) tem sido considerada vantajosa em relação aos demais métodos de determinação de risco para DCV, pois seus ajustes em relação à estatura permitem o estabelecimento de um ponto de corte único e aplicável à população geral, independente de sexo, idade ou etnia ${ }^{10}$.

São muitos os determinantes da obesidade, envolvendo fatores complexos, dentre eles o consumo alimentar, pensando nisso diversos indicadores dietéticos vêm sendo propostos para avaliar a ingestão e a qualidade da dieta, como o Índice de Qualidade da Dieta (IQD), instrumento adaptado para o Brasil baseado na Pirâmide Alimentar ${ }^{11}$, a partir do originalmente americano (Healthy Eating Index), desenvolvido por Kennedy et al. ${ }^{12}$, o qual permite avaliar a ingestão adequada de nutrientes e o número de porções consumidas de todos os grupos alimentares ${ }^{13}$. O IQD se sobressai aos demais métodos devido à possibilidade de observar a dieta de uma forma geral, analisando-se vários componentes e não apenas variáveis dietéticas específicas ${ }^{14}$.

Sabe-se, ainda, que a classe trabalhadora está mais propensa ao desenvolvimento de DCV, uma vez que muitos não realizam atividade física, a própria rotina de trabalho faz com que tenham uma vida mais sedentária e, a grande maioria realiza suas refeições no próprio local de trabalho ${ }^{15}$. Todavia, o local de trabalho vem sendo apontado como possível cenário estratégico para o desenvolvimento de intervenções para a prevenção e controle de peso, objetivando reduzir os riscos de doenças, dentre elas as DCV, melhorar a condição nutricional dos indivíduos, promover melhora da qualidade de vida, aumentar da produtividade e reduzir acidentes de trabalho' ${ }^{16,17}$.

Diante do exposto, o presente estudo objetivou relacionar a qualidade da alimentação de trabalhadores, seu estado nutricional e a relação com risco para DCV.

\section{MÉTODO}

Trata-se de um estudo transversal observacional, realizado com amostra por conveniência de funcionários de uma indústria de comércio de carnes do município de Pitanga, Paraná.

A seleção da amostra foi representativa dos funcionários da empresa, calculada por métodos estatísticos, com base na totalidade de colaboradores. Adotou-se um nível de significância estatística de 95\%, estimando um erro máximo de $\pm 5 \%$.

A coleta de dados ocorreu no período de outubro a dezembro de 2017, em dias agendados pela nutricionista responsável técnica. Os funcionários foram abordados e convidados a participar do estudo, sendo repassadas todas as informações pertinentes à pesquisa e solicitando-se autorização por meio da assinatura do Termo de Consentimento Livre e Esclarecido (TCLE). A coleta de dados foi realizada somente após a aprovação do projeto pelo Comitê de Ética em Pesquisa com Seres Humanos (COMEP) da Universidade Estadual do Centro-Oeste (UNICENTRO), sob parecer de número 2.278.508 de 2017.

Os dados foram obtidos por meio de formulários précodificados, os quais informavam dados gerais, como: idade, estado civil, setor e cargo ocupado, dados socioeconômicos, histórico familiar de DCV, dentre outros.

Para avaliação antropométrica, foi aferido o peso e a estatura, que foram avaliados de acordo com normativas técnicas com uma balança eletrônica com capacidade de 
150 quilos e uma fita inelástica posicionada em parede lisa, sem rodapés. Posteriormente, foi calculado o índice de massa corporal (IMC) e classificado de acordo com o recomendado para adultos ${ }^{18}$.

Para verificação do risco para DCV, foi aferida a circunferência da cintura (CC) e a classificação se deu segundo a Organização Mundial da Saúde, a qual é diferente entre os sexos ${ }^{19}$. Posteriormente, foi realizado o cálculo da RCE utilizando a medida da CC dividida pela altura, ambas em centímetros $(\mathrm{cm})$, com resultado variando de valores próximos de zero (0) a um (1), classificado como risco para DCV valores 0,5 ou mais ${ }^{20}$.

A avaliação da dieta foi realizada por meio do dia alimentar habitual, no qual o indivíduo relatou os alimentos, preparações e bebidas consumidas habitualmente em sua alimentação, com valores de medidas caseiras que posteriormente foram transformados em gramas ou mililitros. Para cálculo e avaliação do consumo alimentar foi utilizado o software Nutrabem ${ }^{\circledR}$, o qual determinou os valores descritivos do IQD, baseados nos grupos alimentares segundo a pirâmide dos alimentos. $O$ IQD possui 12 componentes, sendo 9 grupos alimentares, divididos em dois grupos: "aves, pescados e ovos", "cereais integrais, tubérculos e raízes", "frutas", "hortaliças", "leguminosas", "leites e derivados", "oleaginosas", "óleos e vegetais", grupo de consumo moderado: "açúcares e doces", "carne bovina e suína", "cereais e pães refinados", "gordura de origem animal".

A pontuação de cada componente da dieta variou entre 0 e 10, totalizando 100 pontos como nota máxima. A qualidade da dieta foi dividida em três categorias: de 80 a 100 pontos - dieta saudável, de 50 a 79 pontos - necessita de ajustes no padrão alimentar, menor que 49 pontos dieta inadequada.

Para avaliação da normalidade dos dados foi aplicado o teste de Shapiro Wilk. Para a análise estatística foram calculados valores de frequências, médias e desvio padrão. Em seguida, procedeu-se à associação das variáveis com a análise da variância, seguida de teste de post hoc de Tukey e teste T para amostras independentes. As associações foram testadas em análises bivariadas. Um modelo de regressão logística ordinal de odds proporcional foi utilizado para estimar os valores das razões de chance (RC) e respectivos intervalos de confiança (IC95\%) para cada um dos níveis dos fatores associados à dieta inadequada, simultaneamente, com programa Statistical Package for the Social Sciences, versão 22.0 (SPSS Inc. Chicago, IL, EUA). A significância estatística foi determinada quando o valor de $p<0,05$ foi observado.

\section{RESULTADOS}

Entre os 55 funcionários avaliados, a média de idade foi de 32,2 $\pm 9,9$ anos, houve predominância do sexo masculino $(67,3 \%)$, e a prevalência de etnia branca $92,7 \%(n=51)$. Sobre a escolaridade, a maioria referiu possuir ensino médio completo (43,6\%). Quanto ao estado civil, a maioria $(71,8 \%)$ era casada, com renda familiar mensal média de 2604,00 $\pm 1168,16$ reais (Tabela 1).

Foi observado que indivíduos com sobrepeso apresentaram idade maior em relação os que se encontravam em eutrofia $(p=0,02)$. A RCE e a CC apresentaram valores maiores considerando-se os pontos de cortes estabelecidos na literatura, em indivíduos classificados com obesidade pelo IMC $(p<0,001)$, com valores discrepantes entre eutróficos e obesos.

O IQD médio geral foi de $52,0 \pm 10,9$ pontos, sendo $40 \%(n=22)$ dos funcionários avaliados classificados com dieta inadequada e, 60\% $(n=33)$ deles com necessidade de ajustes na dieta, nenhum dos indivíduos avaliados foi classificado com dieta saudável. Comparada ao estado nutricional, a média de pontos foi menor no grupo com eutrofia $(51,4 \pm 13,3)$.

Ao analisar as variáveis de histórico familiar e pessoal de DCNT, $89,1 \%$ dos trabalhadores referiram algum tipo de histórico familiar e, 21,8\%, histórico pessoal de diabetes mellitus, dislipidemias, DCV e/ou hipertensão arterial sistêmica.

Tabela 1 - Comparação do diagnóstico nutricional com demais variáveis.

\begin{tabular}{|c|c|c|c|c|c|}
\hline \multirow[b]{2}{*}{ Variáveis } & \multirow[b]{2}{*}{ Total $(n=55))$} & \multicolumn{3}{|c|}{ Diagnóstico Nutricional } & \multirow[b]{2}{*}{ Valor de $\mathrm{p}^{\star}$} \\
\hline & & Eutrofia & Sobrepeso & Obesidade & \\
\hline Idade (anos; média/DP) & $32,2 \pm 9,9$ & $27,0 \pm 6,7^{\varepsilon}$ & $35,4 \pm 9,9^{\varepsilon}$ & $31,4 \pm 11,2$ & 0,02 \\
\hline Renda familiar (reais; média/DP) & $2604,0 \pm 1168,2$ & $2426,2 \pm 856,2$ & $2858,6 \pm 1371,4$ & $2150,0 \pm 787,7$ & 0,19 \\
\hline $\mathrm{CC}(\mathrm{cm} ;$ média/DP) & $87,4 \pm 10,5$ & $78,1 \pm 6,2^{\varepsilon}$ & $87,8 \pm 6,9$ & $101,0 \pm 9,5^{£}$ & 0,001 \\
\hline $\mathrm{RCE}$ (média/DP) & $0,5 \pm 0,1$ & $0,4 \pm 0^{\varepsilon}$ & $0,5 \pm 0$ & $0,6 \pm 0,1^{\varepsilon}$ & 0,001 \\
\hline IQD (média/DP) & $52,0 \pm 10,9$ & $51,4 \pm 13,3$ & $52,3 \pm 10,4$ & $52,1 \pm 8,9$ & 0,96 \\
\hline Calorias totais (média/DP) & $1913,3 \pm 756,2$ & $2158,9 \pm 634,7$ & $1817,4 \pm 772,4$ & $1798,4 \pm 867,4$ & 0,31 \\
\hline
\end{tabular}

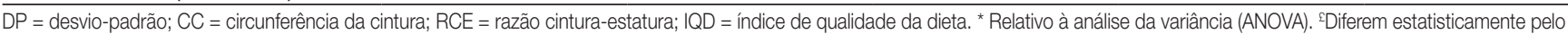
teste post hoc. 
Quanto à avaliação antropométrica, observou-se um IMC médio de $27,05 \pm 4,90 \mathrm{~kg} / \mathrm{m}^{2}$, sendo que $29,1 \%(\mathrm{n}=16)$ encontravam-se eutróficos, $52,7 \%(n=29)$ com sobrepeso e $18,1 \%(n=10)$ com obesidade.

$\mathrm{Na}$ Tabela 2, foi observada a comparação do risco para DCV com algumas variáveis. Verificou-se associação estatisticamente significativa entre as variáveis de idade, $I M C$, calorias totais $(p=0,03 ; p=0,001 ; p=0,05$, respectivamente). Foi observado que, no grupo sem risco para DCV, a idade foi menor em relação ao grupo com risco de DCV $(p=0,03)$. O IMC foi menor no grupo sem risco de DCV $(p=0,001)$. $\bigcirc$ grupo com risco para DCV obteve médias de calorias inferiores quando comparado ao grupo sem risco para DCV $(p=0,05)$. $O I Q D$ apresentou valores maiores no grupo com risco para DCV comparado ao grupo sem risco, porém não foi significativo estatisticamente.

Na Tabela 3, foi comparada a qualidade da dieta pelo IQD com demais variáveis. Houve associação significativa entre dieta inadequada e maior consumo calórico $(p<0,05)$. Na Tabela 4, associando outras variáveis com o $I Q D$, observou-se uma tendência estatística à dieta inadequada entre mulheres e idade, quanto maior a idade, maior tendência a dieta inadequada. O IQD não apresentou associação estatística com as demais variáveis.

Tabela 2 - Comparação do risco cardiovascular com demais variáveis.

\begin{tabular}{lccc}
\hline \multirow{2}{*}{ Variáveis } & \multicolumn{2}{c}{ Risco cardiovascular pela RCE } & Valor de $\mathbf{p}^{*}$ \\
\cline { 2 - 3 } & Sem risco $(\mathbf{n}=\mathbf{2 3})$ & Com risco $(\mathbf{n}=\mathbf{3 2})$ & 0,03 \\
Idade (anos; média/DP) & $27,9 \pm 7,8$ & $35,3 \pm 10,2$ & 0,001 \\
IMC (kg/m²; média/DP) & $23,4 \pm 2,3$ & $29,6 \pm 4,6$ & 0,39 \\
IQD (média/DP) & $50,5 \pm 11,8$ & $53,1 \pm 10,2$ & 0,05 \\
Calorias totais (média/DP) & $2147,2 \pm 703,9$ & $1745,2 \pm 758,2$ & \\
\hline
\end{tabular}

$\mathrm{DP}$ = desvio padrão; IMC = índice de massa corporal; IQD = índice de qualidade da dieta; RCE = razão cintura-estatura; *Relativo ao teste T para amostras independentes.

Tabela 3 - Comparação da qualidade da dieta com demais variáveis.

\begin{tabular}{lccc}
\hline \multirow{2}{*}{ Variáveis } & \multicolumn{2}{c}{ Qualidade da dieta } & \multirow{2}{*}{ Valor de * $^{*}$} \\
\cline { 2 - 3 } & Necessita de ajuste & Dieta inadequada & 0,08 \\
Idade (anos; média/DP) & $34,1 \pm 9,5$ & $29,4 \pm 10,0$ & 0,23 \\
IMC (kg/m; média/DP) & $27,7 \pm 5,1$ & $26,1 \pm 4,5$ & 0,44 \\
CC (cm; média/DP) & $88,2 \pm 11,5$ & $86,1 \pm 9,0$ & 0,20 \\
RCE (média/DP) & $0,5 \pm 0,1$ & $0,5 \pm 0$ & 0,04 \\
Calorias totais (média/DP) & $1743,6 \pm 659,4$ & $2167,9 \pm 833,6$ & \\
\hline
\end{tabular}

$\mathrm{DP}$ = desvio-padrão; CC = circunferência da cintura; IMC = índice de massa corporal; RCE = razão cintura-estatura; * Relativo ao teste T para amostras independentes.

Tabela 4 - Associação entre a presença de dieta inadequada com demais variáveis nutricionais.

\begin{tabular}{|c|c|c|}
\hline \multirow[t]{2}{*}{ Variáveis analisadas } & \multicolumn{2}{|c|}{$\begin{array}{l}\text { Presença de dieta inadequada } \\
\text { pelo Índice de Qualidade da Dieta }\end{array}$} \\
\hline & OR (IC) & Valor de $\mathrm{p}^{*}$ \\
\hline Idade - anos & $1,03(0,96-1,38)$ & 0,07 \\
\hline \multicolumn{3}{|l|}{ Sexo } \\
\hline Feminino & $1,08(0,75-1,29)$ & 0,06 \\
\hline Masculino & 1 & - \\
\hline $\mathrm{IMC}-\mathrm{kg} / \mathrm{m}^{2}$ & $1,15(0,21-2,83)$ & 0,22 \\
\hline \multicolumn{3}{|l|}{ Estado nutricional - IMC } \\
\hline Eutrofia & 1 & - \\
\hline Sobrepeso/obesidade & $0,95(0,68-4,07)$ & 0,51 \\
\hline \multicolumn{3}{|c|}{ Risco para doença cardiovascular pela RCE } \\
\hline Sem risco & 1 & - \\
\hline Com risco & $1,41(0,51-8,12)$ & 0,32 \\
\hline
\end{tabular}

\section{DISCUSSÃO}

Esse estudo buscou traçar um perfil quanto ao risco para DCV em trabalhadores, visando também associar com a qualidade da dieta dos mesmos. Nesse estudo foi observado que o consumo calórico apresentou relação com a qualidade da dieta, ainda a média de calorias totais foi maior na dieta inadequada, isso é devido à densidade energética da dieta, uma alimentação rica em processados, ultraprocessados, açúcares e gorduras no geral são acrescidas de grande quantidade de calorias por grama deficientes ou inadequadas em vitaminas, minerais e demais nutrientes. Essa alimentação é típica da transição nutricional, a chamada ocidentalização da dieta ${ }^{8,21}$.

Na questão do consumo alimentar, inversamente, a média de calorias totais foi maior no grupo sem risco de DCV pelo indicador RCE. É importante destacar aqui que a RCE leva em consideração a CC, a adiposidade central está diretamente relacionada ao consumo de alimentos de alta densidade 
energética, os quais têm facilidade de se acumular nessa região em tecido adiposo, como o grupo sem risco também apresentou valores menores de CC, demonstra que uma dieta pode ter mais calorias pela sua variedade e não somente pela densidade de energia. Sabe-se que uma população com uma dieta mais variada terá uma dieta mais calórica, e não necessariamente uma dieta inadequada, visto que um dos indicadores do IQD leva em consideração a variedade. No que tange à variedade, a dieta da população da população brasileira está se tornando cada vez mais monótona, pobre em frutas e verduras, rica em sódio e bebidas açucaradas, sendo mais acentuada no público masculino. Outro fator importante de ser destacado e avaliado, o qual não foi o foco do presente estudo, é a necessidade de avaliar o gasto energético total, mesmo que estimado por fórmulas, e as calorias ingeridas diariamente $e^{21,22}$.

Em relação à idade, observou-se que, quanto maior a idade, maior é o ganho de peso, bem como risco de desenvolver DCV, de acordo com o indicador RCE. Esta variável também demonstrou tendência a dieta inadequada. Diversos estudos têm comprovado a elevada prevalência de excesso de peso com o passar dos anos em contraste com o baixo peso, evidenciando o fenômeno já citado e conhecido como transição nutricional, tornando a dieta inadequada e o indivíduo propício ao desenvolvimento de DCV e demais doenças crônicas ${ }^{22-24}$.

A RCE demonstrou ser bom indicador do estado nutricional, com valores bem divergentes entre os indivíduos com eutrofia e com obesidade $(p<0,05)$, podendo ser usado como preditor ou complemento de risco de DCV, destacandose em razão do seu ajuste pela estatura, justificando o único valor de referência independente de idade e sexo, além de sua praticidade. Outra vantagem da utilização da RCE se dá pelo fato de considerar a CC, correlacionando-se melhor com a distribuição de gordura comparado ao IMC22,25. Um estudo realizado em Salvador - Bahia, no ano de 2000, com 968 pessoas, sendo 577 mulheres e 391 homens, evidenciou que os indicadores de obesidade abdominal são mais eficazes e estatisticamente significativos. Ao avaliar 1022 adultos atendidos em uma clínica-escola, a RCE mostrou-se significativa no diagnóstico de obesidade e fator de risco ${ }^{26}$.

Apesar de não mediar a composição corporal, sabe-se que o IMC tem forte correlação com a massa de gordura absoluta, associando-se positivamente com o risco de DCV e outras doenças crônicas ${ }^{13}$. No presente estudo, a média do IMC foi de $27,07 \mathrm{~kg} / \mathrm{m}^{2}$, com relação positiva para o risco de $D C V$, resultado muito semelhante foi encontrado em um estudo que avaliou o risco de DCV em profissionais de enfermagem ${ }^{27}$ e, inferior ao encontrado por Vieira et al. ${ }^{13}$, que identificaram IMC médio de $31,38 \mathrm{~kg} / \mathrm{m}^{2}$, essa média mais alta se dá ao fato de ter avaliado indivíduos com excesso de peso.

No que tange o IQD, a média de pontos foi de 52,01 pontos, caracterizando a dieta com necessidades de ajustes, homens obtiverem uma média de pontos superior às mulheres, 52,52 pontos e 50,96 pontos, respectivamente. Do total de funcionários avaliados, $40 \%$ foram classificados com dieta inadequada e $60 \%$ deles necessitavam de ajustes na dieta, nenhum dos indivíduos apresentou dieta adequada. A média do IQD do presente estudo foi semelhante ao estudo que avaliou a qualidade da dieta entre homens e mulheres, com diferença na média entre os $\operatorname{sexos}^{28}$. Ao avaliar a qualidade da dieta de mulheres praticantes de atividade física, observou-se pontuação acima do presente estudo, todavia mais de $90 \%$ da amostra necessitavam de ajustes na dieta $^{29}$. Média de pontos semelhantes ao presente estudo foi encontrada ao avaliar a qualidade da dieta de beneficiários do programa bolsa família, estando mais da metade da população com dieta inadequada ${ }^{30}$.

As mulheres ainda são as que mais sofrerem pressão em relação ao corpo e ao peso, estando frequentemente insatisfeitas com o mesmo, buscando a qualquer custo diversos meios de atingir um padrão já estabelecido, dentre esses meios encontram-se as modificações em seu consumo alimentar, interferindo diretamente na sua qualidade, tornando-se por vezes inadequada, essa tendência à dieta inadequada foi evidenciada no presente estudo ${ }^{30}$. Divergindo com o presente dado, Assumpção et al.$^{28}$ verificaram superioridade na qualidade da dieta feminina, ao analisar a diferença da qualidade da dieta entre homens e mulheres. Um estudo da qualidade da dieta de mulheres climatéricas, Lima et al. ${ }^{31}$ verificaram dieta inadequada e com necessidade de ajustes. Ao analisar a qualidade da dieta de adultos com excesso de peso de uma clínica-escola, sendo a maior parte dos indivíduos estudados do sexo feminino, Vieira et al. ${ }^{13}$ constataram inferioridade na qualidade da dieta de mulheres em relação aos homens, porém o consumo de nutrientes dentro do recomendado. Ao avaliar a qualidade da dieta de mulheres frequentadoras de uma academia, encontrouse média de 57,2 pontos, neste estudo associou-se a baixa qualidade da dieta à condição socioeconômica precária dessas mulheres ${ }^{32}$.

Como pontos fortes com relação a esse trabalho tem-se a avaliação qualitativa da alimentação de trabalhadores e sua associação com risco para DCV. Entretanto, como limitações destacam-se o baixo número amostral e análise do dia alimentar habitual, que pode não trazer um panorama mais fidedigno do padrão alimentar do indivíduo.

\section{CONCLUSÃO}

O risco para DCV, estabelecido pelas medidas e índices antropométricos, apresentaram valores maiores, pelos pontos de cortes estabelecidos na literatura. A maioria da amostra apresentou uma dieta que necessitava de ajustes, sendo que nenhum dos indivíduos possuía uma dieta saudável. Os resultados demonstraram que, com o avançar da idade, os indivíduos tendem a ter uma dieta inadequada, com maior consumo 
calórico e, como consequência, o excesso de peso, tornando-os propícios ao desenvolvimento de DCV e demais morbidades crônicas. Evidenciou-se, também, que as mulheres são o grupo que mais tende a uma dieta inadequada, aumentando o risco de desenvolvimento de doenças crônicas entre as mesmas. Deste modo, faz-se necessário o acompanhamento nutricional, bem como trabalhos de promoção da alimentação adequada e saudável, no âmbito do ambiente de trabalho, uma vez que muitas dessas pessoas não são atingidas por essas atividades. Para melhores resultados, sugere-se mais estudos com intuito de avaliar o gasto energético destes trabalhadores, seu nível de atividade física e relação com a qualidade da dieta, visto que esses dados não foram avaliados no presente estudo.

\section{REFERÊNCIAS}

1. Malta DC, Felisbino-Mendes MS, Machado ÍE, Passos VMA, Abreu DMX, Ishitani LH, et al. Fatores de risco relacionados à carga global de doença do Brasil e Unidades Federadas, 2015. Rev Bras Epidemiol. 2017;20(Suppl 1):217-32.

2. Nespeca M, Cyrillo DC. Avaliação da qualidade da dieta por meio do índice de alimentação saudável de funcionários de uma universidade pública. Nutrire: Rev Soc Bras Alim Nutr. 2010;35(2):81-90.

3. Willett W, Rockstrom J. Dietas saudáveis a partir de sistemas alimentares sustentáveis. Relatório Sumário da Comissão EATLancet. Estocolmo, 2019. [cited 2019 Jun 27]. Available from: $<$ https://eatforum.org/content/uploads/2019/04/EAT-Lancet_ Commission Summary Report Portugese.pdf $>$.

4. Adriouch S, Julia C, Kesse-Guyot E, Méjean C, Ducrot P, Péneau $\mathrm{S}$, et al. Prospective association between a dietary quality index based on a nutrient profiling system and cardiovascular disease risk. Eur J Prev Cardiol. 2016;23(15):1669-76.

5. GBD 2015 Obesity Collaborators, Afshin A, Forouzanfar MH, Reitsma MB, Sur P, Estep K, Lee A, et al. Health effects of overweight and obesity in 195 countries over 25 years. N Engl J Med. 2017;377(1):13-27.

6. Brasil. Ministério da Saúde. Vigitel 2013: vigilância de fatores de risco e proteção para doenças crônicas por inquérito telefônico. Brasília: Ministério da Saúde; 2014. 121p.

7. World Health Organization (WHO). Nutrition. Geneva: World Health Organization; 2019. [cited 2019 Jun 27]. Available from: $<$ https://www.who.int/nutrition/en/>

8. World Health Organization (WHO). Obesity and overweight. Geneva: World Health Organization; 2014.

9. Nguyen DM, El-Serag HB. The epidemiology of obesity. Gastroenterol Clin North Am. 2010;39(1):1-7.

10. Ashwell M, Gunn P, Gibson S. Waist-to-height ratio is a bettes screening tool than waist circumference and BMI for adult cardiometabolic risk factors: systematic review and meta-analysis. Obes Rev. 2012;13(3):275-86.

11. Phillippi ST, Latterza AR, Cruz ATR, Ribeiro LC. Pirâmide alimentar adaptada: guia para escolha dos alimentos. Rev Nutr. 1999;12(1):65-80.

12. Kennedy ET, Ohls J, Carlson S, Fleming K. The Healthy Eating Index: design and applications. J Am Diet Assoc. 1995;95(10):1103-8.

13. Vieira AM, Gomes AS, Vieira RAL, Silva FC, Previato HDRA, Volp ACP. Associação entre medidas antropométricas e de composição corporal com os componentes da síndrome metabólica e índice de qualidade da dieta em adultos com excesso de peso. Demetra. 2016;11(2):399-413.
14. Fisberg RM, Slater B, Barros RR, Lima FD, Cesar CLG, Carandina L, et al. Índice de Qualidade da Dieta: avaliação da adaptação e aplicabilidade. Rev Nutr. 2004;17(3):301-8.

15. Freiberg CK. Associação de fatores de risco para doenças cardiovasculares com qualidade de vida entre trabalhadores. In: Balchiunas D, ed. Gestão de Uan: um resgate do binômio alimentação e nutrição. São Paulo: Roca; 2014. p.27-39.

16. Castro AGP, Bandoni DH, Jaime PC. Promoção da saúde no ambiente de trabalho. In: Balchiunas D, ed. Gestão de Uan: um resgate do binômio alimentação e nutrição. São Paulo: Roca; 2014. p.19-25.

17. Arruda MP, Fujimori E, Lima DB. Avaliação de um programa para promoção do peso saudável entre trabalhadores. J Health Sci Inst. 2016;34(2):82-7.

18. World Health Organization (WHO). Physical status: the use and interpretation of anthropometry. Geneva: World Health Organization; 1995.

19. World Health Organization (WHO). Obesity: preventing and managing the global epidemic. Geneva: World Health Organization; 2000.

20. Pitanga FJG, Lessa I. Razão cintura-estatura como discriminador do risco coronariano de adultos. Rev Assoc Med Bras. 2006;52(3):157-61.

21. Brasil, Ministério da Saúde. Uma análise da situação de saúde e das causas externas. Saúde Brasil. Brasília: Ministério da Saúde; 2014. [cited 2019 Jun 6]. Available from: http://bvsms.saude.gov. br/bvs/publicacoes/saude_brasil_2014_analise_situacao.pdf

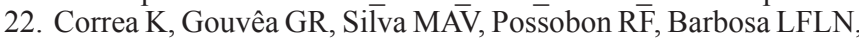
Pereira AC, et al. Qualidade de vida e características dos pacientes diabéticos. Ciência \& Saúde Coletiva. 2017;22(3):921-30.

23. Kümpel DA, Sodré AC, Pomatti DM, Scortegagna HM, Fillipi J, Portella MR, et al. Obesidade em idosos acompanhados pela Estratégia de Saúde da Família. Texto e Contexto Enferm. 2011;20(3):471-7.

24. Oliveira MC, Calahani IM, Previdelli AN, Malta MB, Corrente JE, Papini SJ. Aplicação do Índice de Qualidade da Dieta Revisado antes e após orientação nutricional. Rev Ciênc Med. 2015;24(1):29-36.

25. Haun DR, Pitanga FJG, Lessa I. Razão cintura/estatura comparado a outros indicadores antropométricos de obesidade como preditor de risco coronário elevado. Rev Assoc Med Bras. 2009;55(6):705-11.

26. Sabóia RS, Araújo AP, Barbosa JMA, Galvão CEP, Cruvel JMS, Ferreira SCN. Abdominal obesity and associated factors among adults attending a university health center. Rev Bras Promoç Saúde. 2016;29(2):259-67.

27. Magalhães FJ, Mendonça LBA, Rebouças CBA, Lima FET, Custódio IL, Oliveira SC. Fatores de risco para doenças cardiovasculares em profissionais de enfermagem: estratégias de promoção da saúde. Rev Bras Enferm. 2014;67(3):394-400.

28. Assumpção D, Domene SMA, Fisberg RM, Canesqui AM, Barros MBA. Diferença entre homens e mulheres na qualidade da dieta: estudo de base populacional em Campinas, São Paulo. Ciência e Saúde Coletiva. 2017;22(2):347-58.

29. Costa D, Reis BZ, Vieira DAS, Costa JO, Teixeira PDS, Raposo $\mathrm{OFF}$, et al. Índice de qualidade da dieta de mulheres usuárias de um programa de atividade física regular "Academia da Cidade", Aracajú, SE. Rev Nutr. 2012;25(6):731-41.

30. Lima FEL, Fisberg RM, Uchimura KY, Picheth T. Programa Bolsa-Família: qualidade da dieta de população adulta do município de Curitiba, PR. Rev Bras Epidemiol. 2013;16(1):58-67.

31. Lima LF, Ghetti FF, Lacerda KC, Elias MAR, Silva AA, Luquetti SCPD. Relação entre medidas antropométricas, escolaridade, renda e índice de qualidade da dieta de mulheres climatéricas. HU Rev Juiz de Fora. 2016;42(4):297-305.

32. Horta PM, Santos LC. Qualidade da dieta entre mulheres com excesso de peso atendidas em uma Academia da Cidade de Belo Horizonte, Minas Gerais. Cad Saúde Colet. 2015;23(2):206-13.

Local de realização do estudo: Universidade Estadual do Centro-Oeste, Guarapuava, PR, Brasil.

Conflito de interesse: Os autores declaram não haver. 